\title{
COVID-19 en niños y adolescentes: Mirando un poco más allá de lo físico
}

\section{COVID-19 in children and adolescents: Looking beyond the medical issues}

\author{
Maricarmen Chacín. MD. MSc \\ ${ }^{1}$ Facultad de Ciencias de la Salud, Universidad Simón Bolívar. Barranquilla, Colombia \\ *Dirigir correspondencia a: m.chacin@unisimonbolivar.edu.co
}

Proceso Editorial

Publicado: 221021

DOI 10.17081/innosa.136

CCopyright 2021.

Chacín $\mathrm{M}^{1}$ et al.
La pandemia de COVID-19 ha sido un acontecimiento mundial sin precedentes históricos cercanos. El distanciamiento físico y otras restricciones impuestas por los gobiernos en todo el mundo para contener la propagación del virus han tenido un impacto significativo en todos los habitantes de este planeta, pero probablemente, su influencia en los más jóvenes -niños y adolescentes- ha sido mayor, ya que éstos son más sensibles a la interacción con sus compañeros y a los estímulos sociales que los adultos $(\underline{1}, 2)$. Este nuevo escenario representa una experiencia única en la que la protección de los más vulnerables depende del estricto cumplimiento de las nuevas medidas. Si bien muchas personas se adhieren a las directrices, otras no lo hacen. En particular, está resultando difícil convencer a algunos jóvenes de que se abstengan de reunirse físicamente con sus amigos y de participar en reuniones. Esto quedó claramente demostrado varios informes en que estudiantes que se reunieron en grandes grupos

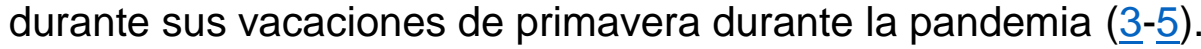

A pesar de esto, los niños y los jóvenes se han visto menos afectados que otros grupos de edad por la infección por coronavirus, pero se han visto desproporcionadamente afectados por los impactos sociales, educativos y económicos de la pandemia $(\underline{6}, \underline{7})$. De hecho, los primeros trabajos indicaron que las tasas de infección en niños y jóvenes eran bajas, de alrededor del alrededor del 1 al $2 \%$ de los casos confirmados de Covid-19, en la mayoría de los países. Para finales de 2020, con la aparición de nuevas variantes más infecciosas del virus se empezó a observar un incremento en la incidencia entre los más, aunado a contar con una mejor infraestructura que mejoró la capacidad diagnóstica inicial, aunque en general, los niños y los jóvenes siempre ha presentado síntomas menos graves que los adultos $(\underline{8}, \underline{9})$. De hecho, en un análisis del primer pico pandémico en el Reino Unido, Ladhani y cols. concluyeron que no hubo un exceso de mortalidad en niños de hasta los 16 años entre enero y mayo de $(\underline{10})$, de forma que solo seis niños menores de 15 años murieron de Covid-19 en el Reino Unido en 2020. 
Medidas como el cierre de escuelas y el distanciamiento físico han tenido probablemente un impacto significativo en los jóvenes, especialmente en los adolescents (11), de forma directa, por la falta de interacción cara a cara con los compañeros, e indirectamente por el aumento del estrés de muchas familias. Aunque las tecnologías digitales han compensado en parte las limitaciones en las relaciones sociales, el distanciamiento físico podría haber tenido un efecto desproporcionado en un grupo de edad para el que la interacción con los compañeros es un medio vital de Desarrollo (11). Además, el encierro ha modificado los hábitos y las rutinas, con un posible impacto a medio y largo plazo en el estilo de vida y el bienestar de los jóvenes (12), y aunque en tiempos de crisis pueda parecer frívolo explorar las experiencias de ocio, las elecciones de ocio realizadas en circunstancias estresantes pueden revelar mucho sobre las estrategias de afrontamiento y las formas de reaccionar ante un cambio radical $(\underline{13}, 14)$.

Es indudable que la investigación sobre los impactos del COVID-19 se ha centrado hasta ahora principalmente en la salud física y en la salud mental. Sin embargo, esta es, hasta cierto punto, una visión simplista de los problemas que aquejan a nuestros jóvenes. Por ejemplo, los jóvenes que ya se enfrentan problemas de magnitud en sus vidas (como los que viven en la pobreza o con una discapacidad) parecen haber sido más afectados por la pandemia, y son de gran preocupación aquellos efectos que veremos a largo plazo ocasionados por limitaciones educativas y laborales. Por ejemplo, aproximadamente la mitad de los hogares han sufrido algún tipo de interrupción relacionada con el trabajo y según la Organización para la Cooperación y el Desarrollo Económicos (OCDE), el 51\% de los jóvenes de 18 a 29 años (de forma personal o un familiar cercano) han sufrido interrupciones relacionadas con el trabajo desde el comienzo de la pandemia de COVID-19 en forma de pérdida de empleo, uso de un plan de retención de empleo, reducción de la jornada laboral y/o recorte de sueldo (15). Los jóvenes de las clases sociales más bajas se han visto más afectados. Uno de cada cinco jóvenes que dicen pertenecer a las clases bajas o trabajadoras declaran haber perdido su empleo, frente a sólo uno de cada ocho de los que dicen pertenecer a la clase media. Incluso en los países en los que las tasas de pérdida de empleo y las dificultades financieras relacionadas eran relativamente bajas, los jóvenes están preocupados. El $63 \%$ de los jóvenes de los países de la OCDE están preocupados por las finanzas de su hogar y el bienestar social y económico general. Aunque este porcentaje es ligeramente inferior al de todos los grupos de edad juntos (67\%), sigue siendo una mayoría alarmante de jóvenes (15).

Nuestra región no ha podido escapar a esta desoladora tendencia pues 1 de cada 6 personas de entre 18 y 29 años ha dejado el trabajo desde que comenzó la pandemia, lo que obligó a muchos a abandonar sus estudios. La precariedad del empleo para los jóvenes aumentó en toda la region (16) de forma que los sentimientos de tristeza, miedo y ansiedad, así como el desánimo tras la crisis prevalecen entre los jóvenes, donde una proporción mayor ha visto afectado su bienestar y salud mental. Si bien la pérdida de empleos entre los jóvenes causó interrupciones en los programas de educación y capacitación, el colapso de las empresas y la caída de los salarios aumentaron la precariedad, el subempleo y el trabajo informal. Esto sin dudas nos traerá, a mediano plazo, más inequidad, menos movilidad social, menor acceso a una buena alimentación y a servicios de salud de calidad, Ilevándonos a plantear la posibilidad que esta catástrofe nos ponga frente a la pérdida de una generación completa, por lo cual éste es el momento indicado para implementar medidas para prevenir esta situacion a futuro. 


\section{REFERENCIAS}

1. L L, H R, R C, Y H, Z Q, C L, et al. The Effect of COVID-19 on Youth Mental Health. Psychiatr Q [Internet]. 2020 Sep 1 [cited 2021 Oct 25];91(3):841-52. Available from: https://pubmed.ncbi.nlm.nih.gov/32319041/

2. N C-B, W R, SE K, AJ R. Adolescent Adjustment During COVID-19: The Role of Close Relationships and COVID-19-related Stress. J Res Adolesc [Internet]. 2021 Sep 1 [cited 2021 Oct 25];31(3):608-22. Available from: https://pubmed.ncbi.nlm.nih.gov/34448310/

3. Wilson E, Donovan C V, Campbell M, Chai T, Pittman K, Seña AC, et al. Multiple COVID-19 Clusters on a University Campus - North Carolina, August 2020. Morb Mortal Wkly Rep [Internet]. 2020 Oct 2 [cited 2021 Oct 25];69(39):1416. Available from: /pmc/articles/PMC7537562/

4. Vang KE. Participation in Fraternity and Sorority Activities and the Spread of COVID-19 Among Residential University Communities - Arkansas, August 21-September 5, 2020. MMWR Morb Mortal Wkly Rep [Internet]. 2021 Jan 8 [cited 2021 Oct 25];70(1):20-3. Available from: https://www.cdc.gov/mmwr/volumes/70/wr/mm7001a5.htm

5. Nieto D, Nieto I. Estilo de vida de estudiantes de la Universidad del Atlántico: un estudio descriptivo-transversal. Ciencia e Innovación en Salud. 2020. e68:19-30 DOI 10.17081/innosa.69

6. Campos MM, Vieira LF. COVID-19 and early childhood in Brazil: impacts on children's wellbeing, education and care. https://doi.org/101080/1350293X20211872671 [Internet]. 2021 [cited 2021 Oct 25];29(1):125-40. Available from: https://www.tandfonline.com/doi/abs/10.1080/1350293X.2021.1872671

7. COVID-19 guidelines for ECEC services [Internet]. [cited 2021 Oct 25]. Available from: https://education.nsw.gov.au/early-childhood-education/coronavirus/advice-for-servicesand-providers

8. Knight SR, Ho A, Pius R, Buchan I, Carson G, Drake TM, et al. Risk stratification of patients admitted to hospital with covid-19 using the ISARIC WHO Clinical Characterisation Protocol: development and validation of the 4C Mortality Score. BMJ [Internet]. 2020 Sep 9 [cited 2021 Oct 25];370:22. Available from: https://www.bmj.com/content/370/bmj.m3339

9. F G, B S-G, A N-J, M L, L L, FI CC, et al. COVID-19 in children and adolescents in Europe: a multinational, multicentre cohort study. Lancet Child Adolesc Heal [Internet]. 2020 Sep 1 [cited 2021 Oct 25];4(9):653-61. Available from: https://pubmed.ncbi.nlm.nih.gov/32593339/

10. Smith C, Odd D, Harwood R, Ward J, Linney M, Clark M, et al. Deaths in Children and Young People in England following SARS-CoV-2 infection during the first pandemic year: a national study using linked mandatory child death reporting data. 2021 Jul 7 [cited 2021 Oct 25]; Available from: https://www.researchsquare.com

11. A O, L T, SJ B. The effects of social deprivation on adolescent development and mental health. Lancet Child Adolesc Heal [Internet]. 2020 Aug 1 [cited 2021 Oct 25];4(8):634-40. Available from: https://pubmed.ncbi.nlm.nih.gov/32540024/

12. Ventura PS, Ortigoza AF, Castillo Y, Bosch Z, Casals S, Girbau C, et al. Children's health habits and covid-19 lockdown in catalonia: Implications for obesity and non-communicable 
diseases. Nutrients. 2021 May 1;13(5).

13. Liu H-L (Stella), Lavender-Stott ES, Carotta CL, Garcia AS. Leisure experience and participation and its contribution to stress-related growth amid COVID-19 pandemic. https://doi.org/101080/0261436720211942526 [Internet]. 2021 [cited 2021 Oct 25]; Available from: https://www.tandfonline.com/doi/abs/10.1080/02614367.2021.1942526

14. Codina N, Pestana J V., Stebbins RA. Serious and casual leisure activities in the construction of young adult identity: A study based on participants' self-descriptions. OBETS. 2017;12(Extra1):65-80.

15. Risks that matter 2020: The long reach of COVID-19 [Internet]. [cited 2021 Oct 25]. Available from: https://www.oecd.org/coronavirus/policy-responses/risks-that-matter-2020-the-longreach-of-covid-19-44932654/

16. Unidas N, Nations U. ECLAC/ILO No. 23: Employment Situation in Latin America and the Caribbean. Employment trends in an unprecedent crisis: policy challenges. 2020; 